\title{
DETERMINATION OF COUPLED SWAY, ROLL, AND YAW MOTIONS OF A FLOATING BODY IN REGULAR WAVES
}

\author{
S. N. DAS and S. K. DAS
}

Received 21 May 2003

\begin{abstract}
This paper investigates the motion response of a floating body in time domain under the influence of small amplitude regular waves. The governing equations of motion describing the balance of wave-exciting force with the inertial, damping, and restoring forces are transformed into frequency domain by applying Laplace transform technique. Assuming the floating body is initially at rest and the waves act perpendicular to the vessel of lateral symmetry, hydrodynamic coefficients were obtained in terms of integrated sectional addedmass, damping, and restoring coefficients, derived from Frank's close-fit curve. A numerical experiment on a vessel of 19190 ton displaced mass was carried out for three different wave frequencies, namely, $0.56 \mathrm{rad} / \mathrm{s}, 0.74 \mathrm{rad} / \mathrm{s}$, and $1.24 \mathrm{rad} / \mathrm{s}$. The damping parameters $\left(\varsigma_{i}\right)$ reveal the system stability criteria, derived from the quartic analysis, corresponding to the undamped frequencies $\left(\beta_{i}\right)$. It is observed that the sway and yaw motions become maximum for frequency $0.56 \mathrm{rad} / \mathrm{s}$, whereas roll motion is maximum for frequency $0.74 \mathrm{rad} / \mathrm{s}$. All three motions show harmonic behavior and attain dynamic equilibrium for time $t>100$ seconds. The mathematical approach presented here will be useful to determine seaworthiness characteristics of any vessel when wave amplitudes are small and also to validate complex numerical models.
\end{abstract}

2000 Mathematics Subject Classification: 34C15, 70K42, 70K25.

1. Introduction. Precise prediction of hydrodynamic behavior and motion response of a floating body in water waves is essential for proper harbor design. A floating body excited by waves experiences six degrees of motion constituting three translatory and three rotational motions. These motions can be described kinematically in terms of surge, sway, and heave, which are translations along the $x-, y$-, and $z$-axes, and rotations about the same set of axes are roll, pitch, and yaw (Figure 1.1). For a floating body with lateral symmetry in shape and weight distribution, the six coupled equations of motion can be reduced to two sets of equations, where the first set consisting of surge, heave, and pitch can be decoupled from the second set consisting of sway, roll, and yaw. We investigate the second set since the roll motion is important with respect to the stability of the floating body.

Important investigations to understand the hydrodynamic behavior and motion response of a floating body were first started by W. Froude with an initial study of rolling. Subsequently, several investigations were carried out with the development of strip theory in ship hydrodynamics, in conjunction with the study of ship vibration. In most of the studies, ship motions were considered in a calm water environment until the landmark research work of Weinblum and St. Denis [10], which takes into account 


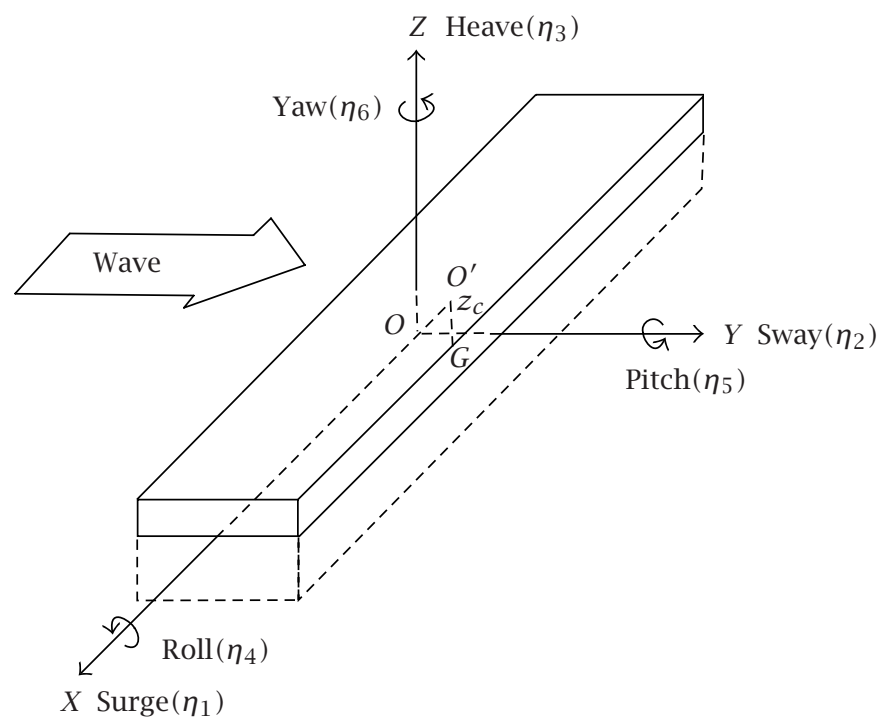

FIGURE 1.1. Sign convention for translatory and angular displacements of a floating body with the direction of wave propagation.

sea environment. Cummins [1] formulated linearized equations of motion of a floating body in transient seaway. Tasai [6] introduced a strip theory to calculate sway-roll-yaw motions for a ship in oblique waves with zero forward speed. Vugts [9] reported experimental observations on sway and roll amplitudes for various cylinder shapes in beam waves. Salvesen et al. [4] presented a new strip theory for predicting heave, pitch, sway, roll, and yaw motions as well as wave-induced vertical and horizontal shear forces, bending moments, and torsional moments for a ship in arbitrary heading waves with constant forward speed. The motion of a floating horizontal cylinder in a uniform inviscid fluid at irregular wave frequencies was studied by Ursell [8], considering it as a classical potential flow problem. Mulk and Falzarano [3] studied nonlinear ship rolling motion in six degrees of freedom by using numerical path-following techniques and numerical integration. Faltinsen et al. [2] studied nonlinear wave loads on a vertical cylinder. The effect of nonlinear damping and restoring in ship rolling and ship stability in dynamic environments can be found in the recent works of Taylan [7] and Surendran and Reddy [5].

The present study attempts to develop a mathematical approach to determine sway, roll, and yaw motions and considers the general formulation given by Salvesen et al. [4] to describe harmonic response of a floating body. This approach is useful for three main purposes: (i) to get insight into the effect of various parameters and its relative importance while wave forces act in concurrence, (ii) to validate complex numerical models by providing useful benchmarking, and (iii) to provide accurate mathematical tools to supplement detailed model testing.

2. Problem formulation. Let $(x, y, z)$ be a right-handed coordinate system fixed with respect to the mean position of the body and $z$-axis considered vertically upward. The 
origin $O$ lies in the undisturbed free surface. Let the translatory displacements in $x, y$, and $z$ directions with respect to the origin be $\eta_{1}, \eta_{2}$, and $\eta_{3}$ indicating surge, sway, and heave, respectively. In rotational motion, the angular displacements about the same set of axes are $\eta_{4}, \eta_{5}$, and $\eta_{6}$ indicating roll, pitch, and yaw, respectively. The definition of six motions of any floating body with sign convention is shown in Figure 1.1.

In order to construct the governing equations of motion, the following assumptions are made.

(i) The floating body is slender and rigid with symmetric distribution of mass.

(ii) Motion amplitude is small so that equations can be linearized.

(iii) Except in roll motion, the effect of viscosity is neglected.

(iv) Incident waves are unidirectional and of single periodicity.

(v) Due to lateral symmetry, longitudinal and transverse motions are decoupled.

Under the above assumptions, six linearly coupled differential equations of motions can be written as (see [4])

$$
\sum_{k=1}^{6}\left[\left(M_{j k}+A_{j k}\right) \ddot{\eta}_{k}+B_{j k} \dot{\eta}_{k}+C_{j k} \eta_{k}\right]=F_{j}, \quad j=1,2, \ldots, 6
$$

where $M_{j k}$ are the components of the generalized mass matrix of the ship, $A_{j k}$ and $B_{j k}$ are the frequency-dependent added-mass and damping coefficients, respectively, $C_{j k}$ are the hydrostatic restoring coefficients, and $F_{j}$ are the external exciting forces or moments. The generalized form of mass matrix coefficient is given by

$$
M_{j k}=\left[\begin{array}{cccccc}
M & 0 & 0 & 0 & M z_{c} & 0 \\
0 & M & 0 & -M z_{c} & 0 & 0 \\
0 & 0 & M & 0 & 0 & 0 \\
0 & -M z_{c} & 0 & I_{4} & 0 & -I_{46} \\
M z_{c} & 0 & 0 & 0 & I_{5} & 0 \\
0 & 0 & 0 & -I_{46} & 0 & I_{6}
\end{array}\right]
$$

where $M$ is the mass of the floating body, $I_{j}$ is the moment of inertia in the $j$ th mode of motion, and $I_{j k}$ is the product of inertia for the $k$ th mode of motion coupled with the $j$ th mode. The coordinate of the center of gravity $G$ is $\left(O^{\prime}, 0, z_{c}\right)$, where $O^{\prime}$ and $z_{c}$ are the $x$-coordinate and the $z$-coordinate of the center of gravity, respectively (Figure 1.1). The added-mass and damping coefficient matrices are expressed as

$$
A_{j k}=\left[\begin{array}{cccccc}
A_{11} & 0 & A_{13} & 0 & A_{15} & 0 \\
0 & A_{22} & 0 & A_{24} & 0 & A_{26} \\
A_{31} & 0 & A_{33} & 0 & A_{35} & 0 \\
0 & A_{42} & 0 & A_{44} & 0 & A_{46} \\
A_{51} & 0 & A_{53} & 0 & A_{55} & 0 \\
0 & A_{62} & 0 & A_{64} & 0 & A_{66}
\end{array}\right], \quad B_{j k}=\left[\begin{array}{cccccc}
B_{11} & 0 & B_{13} & 0 & B_{15} & 0 \\
0 & B_{22} & 0 & B_{24} & 0 & B_{26} \\
B_{31} & 0 & B_{33} & 0 & B_{35} & 0 \\
0 & B_{42} & 0 & B_{44} & 0 & B_{46} \\
B_{51} & 0 & B_{53} & 0 & B_{55} & 0 \\
0 & B_{62} & 0 & B_{64} & 0 & B_{66}
\end{array}\right] .
$$


3. Sway, roll, and yaw motions. Following the assumptions, sway, roll, and yaw motions are described as

$$
\left\{\left[M_{i j}\right]+\left[A_{i j}\right]\right\}\left[\ddot{\eta}_{i}\right]+\left[B_{i j}\right]\left[\dot{\eta}_{i}\right]+\left[C_{i j}\right]\left[\eta_{i}\right]=\left[F_{j}\right]
$$

where

$$
\begin{aligned}
& {\left[M_{i j}\right]=} {\left[\begin{array}{ccc}
M & -M z_{c} & 0 \\
-M z_{c} & I_{4} & -I_{46} \\
0 & -I_{46} & I_{6}
\end{array}\right], \quad\left[A_{i j}\right]=\left[\begin{array}{lll}
A_{22} & A_{24} & A_{26} \\
A_{42} & A_{44} & A_{46} \\
A_{62} & A_{64} & A_{66}
\end{array}\right], } \\
& {\left[B_{i j}\right]=} {\left[\begin{array}{lll}
B_{22} & B_{24} & B_{26} \\
B_{42} & B_{44} & B_{46} \\
B_{62} & B_{64} & B_{66}
\end{array}\right], \quad\left[C_{i j}\right]=\left[\begin{array}{ccc}
0 & 0 & 0 \\
0 & C_{44} & 0 \\
0 & 0 & 0
\end{array}\right], } \\
& {\left[\ddot{\eta}_{i}\right]=\left[\begin{array}{l}
\ddot{\eta}_{2} \\
\ddot{\eta}_{4} \\
\ddot{\eta}_{6}
\end{array}\right], \quad\left[\dot{\eta}_{i}\right]=\left[\begin{array}{l}
\dot{\eta}_{2} \\
\dot{\eta}_{4} \\
\dot{\eta}_{6}
\end{array}\right], \quad\left[\eta_{i}\right]=\left[\begin{array}{c}
\eta_{2} \\
\eta_{4} \\
\eta_{6}
\end{array}\right], \quad\left[F_{j}\right]=\left[\begin{array}{l}
F_{2} \\
F_{4} \\
F_{6}
\end{array}\right], }
\end{aligned}
$$

$\dot{\eta}_{i}$ and $\ddot{\eta}_{i}$ being the velocity and acceleration in the $i$ th mode of motion, respectively. Substituting (3.2) in (3.1), the governing equations are obtained as

$$
\begin{aligned}
& \left(A_{22}+M\right) \ddot{\eta}_{2}+B_{22} \dot{\eta}_{2}+\left(A_{24}-M z_{c}\right) \ddot{\eta}_{4}+B_{24} \dot{\eta}_{4}+A_{26} \ddot{\eta}_{6}+B_{26} \dot{\eta}_{6}+=F_{2}, \\
& \left(A_{42}-M z_{c}\right) \ddot{\eta}_{2}+B_{42} \dot{\eta}_{2}+\left(A_{44}+I_{4}\right) \ddot{\eta}_{4}+B_{44} \dot{\eta}_{4}+C_{44} \eta_{4}+\left(A_{46}-I_{46}\right) \ddot{\eta}_{6}+B_{46} \dot{\eta}_{6}=F_{4}, \\
& A_{62} \ddot{\eta}_{2}+B_{62} \dot{\eta}_{2}+\left(A_{64}-I_{46}\right) \ddot{\eta}_{4}+B_{64} \dot{\eta}_{4}+\left(A_{66}+I_{6}\right) \ddot{\eta}_{6}+B_{66} \dot{\eta}_{6}=F_{6} .
\end{aligned}
$$

Considering the forward speed equal to zero, the expressions for added-mass and damping coefficients are given in the appendix. The moment of inertia $\left(I_{j}\right)$ and the product of inertia $\left(I_{j k}\right)$ can be determined for any particular floating body. The restoring coefficient $C_{44}$ which appears in the roll equation (3.4) can be expressed as

$$
C_{44}=\rho g \nabla \bar{G} \bar{M},
$$

where $\nabla$ is the displaced volume of the body in calm water, $\bar{G} \bar{M}$ is the metacentric height, $\rho$ is the mass density of water, and $g$ is the gravitational acceleration. The wave exciting forces and moments are

$$
F_{i}=\bar{F}_{i} \sin (\varpi t+\varepsilon), \quad i=2,4,6,
$$

where $\bar{F}_{2}, \bar{F}_{4}$, and $\bar{F}_{6}$ are the amplitudes of the sway exciting force, roll exciting moment, and yaw exciting moment, respectively, and $\varepsilon$ is the phase angle. $\varpi$ represents the encountering frequency. Since there is no forward speed of the body, the amplitudes of sway exciting force, roll exciting moments, and yaw exciting moments can be obtained 
as (see [4])

$$
\begin{aligned}
& \bar{F}_{2}=\alpha \rho \int\left(f_{2}+h_{2}\right) d \xi, \\
& \bar{F}_{4}=\alpha \rho \int\left(f_{4}+h_{4}\right) d \xi, \\
& \bar{F}_{6}=\alpha \rho \int \xi\left(f_{2}+h_{2}\right) d \xi,
\end{aligned}
$$

where $\alpha$ is the amplitude of the incident wave, $f_{i}$ and $h_{i}$ represent the sectional FroudeKriloff force and sectional diffraction force, respectively, and $\xi$ is a variable of integration in $x$ direction. The integration has been taken over the length of the body.

4. Method of solution. The coupled sway, roll, and yaw motions can be rewritten in the following form after normalizing by the respective coefficient of the acceleration term:

$$
\begin{gathered}
\ddot{\eta}_{2}+a_{2} \dot{\eta}_{2}+b_{1} \ddot{\eta}_{4}+b_{2} \dot{\eta}_{4}+c_{1} \ddot{\eta}_{6}+c_{2} \dot{\eta}_{6}=K_{2} \sin \varpi t, \\
a_{4} \ddot{\eta}_{2}+a_{5} \dot{\eta}_{2}+\ddot{\eta}_{4}+b_{5} \dot{\eta}_{4}+b_{6} \eta_{4}+c_{4} \ddot{\eta}_{6}+c_{5} \dot{\eta}_{6}=K_{4} \sin \varpi t, \\
a_{7} \ddot{\eta}_{2}+a_{8} \dot{\eta}_{2}+b_{7} \ddot{\eta}_{4}+b_{8} \dot{\eta}_{4}+\ddot{\eta}_{6}+c_{8} \dot{\eta}_{6}=K_{6} \sin \varpi t .
\end{gathered}
$$

For a given frequency, the added-mass and damping coefficients are considered constant for small amplitude motion. In the absence of a phase angle, we set $\varepsilon=0$. The Laplace transform of (4.1) gives

$$
l_{i} f_{2}(s)+m_{i} f_{4}(s)+n_{i} f_{6}(s)=r_{j} \quad(i=1, \ldots, 3, j=1, \ldots, 4),
$$

where

$$
f_{i}(s)=\int_{0}^{\infty} e^{-s t} \eta_{i}(t) d t
$$

The expressions for $l_{i}, m_{i}, n_{i}$, and $r_{j}$ are given in the appendix. Equation (4.2) contains three unknowns, namely, $f_{2}(s), f_{4}(s)$, and $f_{6}(s)$, which can be solved in the frequency domain by using Cramer's rule:

$$
f_{2}(s)=\frac{D_{1}}{D}, \quad f_{4}(s)=\frac{D_{2}}{D}, \quad f_{6}(s)=\frac{D_{3}}{D},
$$

where

$$
\begin{array}{rlr}
D_{1}=\left|\begin{array}{lll}
r_{1} & m_{1} & n_{1} \\
r_{2} & m_{2} & n_{2} \\
r_{3} & m_{3} & n_{3}
\end{array}\right|, & D_{2}=\left|\begin{array}{lll}
l_{1} & r_{1} & n_{1} \\
l_{2} & r_{2} & n_{2} \\
l_{3} & r_{3} & n_{3}
\end{array}\right|, \\
D_{3}=\left|\begin{array}{lll}
l_{1} & m_{1} & r_{1} \\
l_{2} & m_{2} & n_{2} \\
l_{3} & m_{3} & n_{3}
\end{array}\right|, & D=\left|\begin{array}{lll}
l_{1} & m_{1} & n_{1} \\
l_{2} & m_{2} & n_{2} \\
l_{3} & m_{3} & n_{3}
\end{array}\right| .
\end{array}
$$

To evaluate (4.4), the denominator term $D$ should be nonsingular, that is, $D \neq 0$. Now expanding determinant $D$, we obtain

$$
D=l_{1}\left(m_{2} n_{3}-n_{2} m_{3}\right)+l_{2}\left(m_{3} n_{1}-n_{3} m_{1}\right)+l_{3}\left(m_{1} n_{2}-n_{1} m_{2}\right) .
$$


Substituting the expressions for $l_{i}, m_{i}$, and $n_{i}$ in (4.6), one can write

$$
D=g_{4} s^{2}\left(s^{4}+r_{3} s^{3}+r_{2} s^{2}+r_{1} s+r_{0}\right)=g_{4} s^{2} Q
$$

where $Q$ is the quartic with respect to the frequency-dependent variable $s$. Expressing $Q$ as the product of two quadratic factors, we further write

$$
Q=\left(s^{2}+2 \varsigma_{1} \beta_{1} s+\beta_{1}^{2}\right)\left(s^{2}+2 \varsigma_{2} \beta_{2} s+\beta_{2}^{2}\right) .
$$

Similarly, determinant $D_{1}$ in (4.5) can be expressed as (see the appendix)

$$
D_{1}=r_{1}\left(m_{2} n_{3}-n_{2} m_{3}\right)+r_{2}\left(m_{3} n_{1}-n_{3} m_{1}\right)+r_{3}\left(m_{1} n_{2}-n_{1} m_{2}\right)=\frac{s \sum_{i=0}^{6} D_{i}^{1} s^{i}}{\left(s^{2}+\varpi^{2}\right)},
$$

where $D_{i}^{1}=f\left\{a_{i}, b_{i}, c_{i}, \varpi, K_{i}, \eta_{2 i}(0), \dot{\eta}_{2 i}(0)\right\}$. Substituting (4.7), (4.8), and (4.9) in (4.4), we obtain

$$
f_{2}(s)=\frac{s \sum_{i=0}^{6} D_{i}^{1} s^{i}}{g_{4} s^{2}\left(s^{2}+\varpi^{2}\right)\left(s^{2}+2 \varsigma_{1} \beta_{1} s+\beta_{1}^{2}\right)\left(s^{2}+2 \varsigma_{2} \beta_{2} s+\beta_{2}^{2}\right)} .
$$

The partial fraction (4.10) gives

$$
f_{2}(s)=\frac{1}{g_{4}}\left[\frac{\alpha_{1}}{s}+\frac{\alpha_{2} s+\alpha_{3}}{s^{2}+\varpi^{2}}+\frac{\alpha_{4} s+\alpha_{5}}{s^{2}+2 \varsigma_{1} \beta_{1} s+\beta_{1}^{2}}+\frac{\alpha_{6} s+\alpha_{7}}{s^{2}+2 \varsigma_{2} \beta_{2} s+\beta_{2}^{2}}\right],
$$

where $\alpha_{i}=f\left\{D_{i}^{1}, \varpi, \beta_{i}, \varsigma_{i}\right\}$. Similarly, the expressions for $f_{4}(s)$ and $f_{6}(s)$ are

$$
\begin{aligned}
& f_{4}(s)=\frac{1}{g_{4}}\left[\frac{\alpha_{1}^{\prime} s+\alpha_{2}^{\prime}}{s^{2}+\varpi^{2}}+\frac{\alpha_{3}^{\prime} s+\alpha_{4}^{\prime}}{s^{2}+2 \varsigma_{1} \beta_{1} s+\beta_{1}^{2}}+\frac{\alpha_{5}^{\prime} s+\alpha_{6}^{\prime}}{s^{2}+2 \varsigma_{2} \beta_{2} s+\beta_{2}^{2}}\right], \\
& f_{6}(s)=\frac{1}{g_{4}}\left[\frac{\alpha_{1}^{\prime \prime}}{s}+\frac{\alpha_{2}^{\prime \prime} s+\alpha_{3}^{\prime \prime}}{s^{2}+\varpi^{2}}+\frac{\alpha_{4}^{\prime \prime} s+\alpha_{5}^{\prime \prime}}{s^{2}+2 \varsigma_{1} \beta_{1} s+\beta_{1}^{2}}+\frac{\alpha_{6}^{\prime \prime} s+\alpha_{7}^{\prime \prime}}{s^{2}+2 \varsigma_{2} \beta_{2} s+\beta_{2}^{2}}\right],
\end{aligned}
$$

where $\alpha_{i}, \alpha_{i}^{\prime}$, and $\alpha_{i}^{\prime \prime}$ are unknown coefficients required to be determined. Equating the like powers of $s$, a set of linear algebraic equations involving the above unknown coefficients are obtained which are then solved by using the Gauss elimination method.

5. Numerical experiment. To get an insight into the effect of various parameters on sway, roll, and yaw motions, a ship of length $=150 \mathrm{~m}$, beam $=20.06 \mathrm{~m}$, draught $=$ $9.88 \mathrm{~m}$, and mass $=19190$ tons was assumed, for which the beam-draft ratio becomes nearly equal to two. The location of center of gravity $G$ is considered at the point $O^{\prime}$, which is $-1 \mathrm{~m}$ away from the origin $O$. The monochromatic sinusoidal waves act perpendicular to the longitudinal axis of the ship with three different frequencies of $0.56 \mathrm{rad} / \mathrm{s}$, $0.74 \mathrm{rad} / \mathrm{s}$, and $1.24 \mathrm{rad} / \mathrm{s}$, corresponding to the wave height of $1.0 \mathrm{~m}$. The coefficients related to sectional added mass, sectional damping, and sectional wave exciting force were used from the experimental results of Vugts [9] and Frank's close-fit curve (Table 5.1), for a cylinder with rectangular cross-section with identical beam-draft ratio.

A computer program, "SIPCOEF," was developed to generate the relevant coefficients corresponding to the set of linear equations for each mode of motion. In order to obtain 
DETERMINATION OF COUPLED SWAY, ROLL, AND YAW MOTIONS ...

TABLE 5.1. Computed sectional coefficients of the floating body.

\begin{tabular}{l|lccc}
\hline \multirow{2}{*}{ Wave } & Frequency $(\mathrm{Rad} / \mathrm{s})$ & 0.56 & 0.74 & 1.24 \\
\cline { 2 - 5 } & Period $(\mathrm{s})$ & 11.2 & 8.5 & 5.1 \\
\hline \multirow{5}{*}{ Sectional } & Sway added mass & 1.6 & 0.65 & 0.05 \\
coefficients & Roll added mass & 0.07 & 0.055 & 0.035 \\
& Sway-roll added mass & -0.25 & -0.13 & -0.02 \\
& Sway damping & 0.6 & 1.0 & 0.7 \\
& Roll damping & 0.01 & 0.02 & 0.012 \\
& Sway-roll damping & -0.07 & -0.16 & -0.1 \\
& Sway exciting force & 2.25 & 1.5 & 0.34 \\
& Roll exciting moment & 1.9 & 1.2 & 0.28 \\
\hline
\end{tabular}

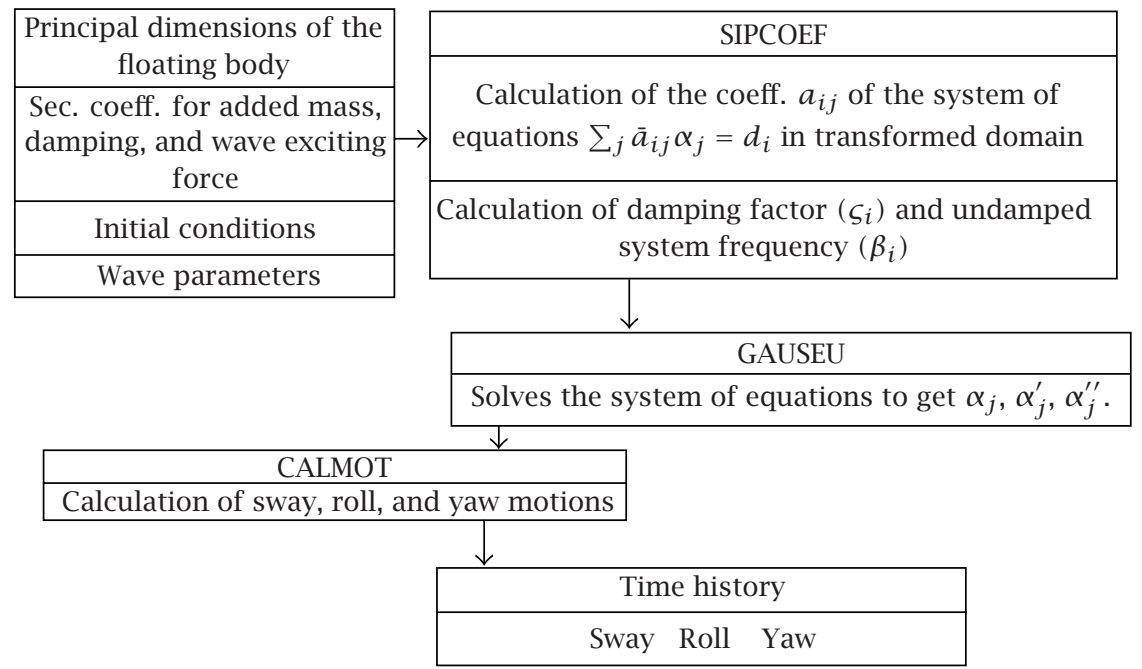

FIGURE 5.1. Schematic diagram of the mathematical model development.

unknown coefficients in the transformed domain, the Gauss elimination method was used. A computer program, "GAUSEU," was used to solve the system of equations. Finally, the time evolution of sway, roll, and yaw motions for a particular wave frequency was obtained from the program "CALMOT." The schematic diagram of the mathematical model development is shown in Figure 5.1.

6. System stability and quartic analysis. In order to obtain the inverse of the Laplace transform, determinant $D$ which appears as the denominator is set equal to zero. This leads to the condition that either $Q$ or $g_{4} s^{2}$ is equal to zero. For $Q=0$, the roots of the characteristic equation are obtained in the frequency domain. The variables $\varsigma_{i}$ and $\beta_{i}(i=1,2)$ which appear in the biquadratics are known as damping factors and undamped natural frequencies of the damped system, respectively. As the factor $g_{4} s^{2}$ is 
TABLE 6.1. Computed damping coefficients.

\begin{tabular}{c|c|c}
\hline \multirow{2}{*}{$\begin{array}{c}\text { Wave frequency } \\
(\mathrm{rad} / \mathrm{s})\end{array}$} & \multicolumn{2}{|c}{ Damping coefficients } \\
\cline { 2 - 3 } & \multicolumn{1}{|c}{$\mathrm{S}_{2}$} \\
\hline 0.56 & 1.13 & 0.053 \\
0.74 & 1.29 & 0.04 \\
1.24 & 2.3 & 0.077 \\
\hline
\end{tabular}

set equal to zero in $D$, the corresponding $\varsigma_{3}$ also becomes zero. This manifests pure oscillation, without damping or building up. As the numerical values for $\varsigma_{i}$ are of prime importance, their role on system stability is summarized as follows:

(i) $\varsigma_{i} \leq-1$ implies that the motion will be a nonoscillatory divergence;

(ii) $-1<\varsigma_{i}<0$ implies that the motion will be a divergent oscillation;

(iii) $0<\varsigma_{i}<1$ implies that the motion will be oscillatory and will die out;

(iv) $\varsigma_{i} \geq 1$ implies that the motion will die out without oscillation;

(v) $\varsigma_{i}=0$ implies that the motion will be oscillatory.

Once the quadratic factors are obtained, the corresponding oscillatory/nonoscillatory features are determined depending upon the values of $\varsigma_{i}$. The frequency-wise variation of damping coefficients, $\varsigma_{1}$ and $\varsigma_{2}$, is given in Table 6.1.

If $\beta_{i d}$ are damped natural frequencies, their relations with undamped natural frequencies can be expressed as $\beta_{1 d}=\beta_{1} \sqrt{\varsigma_{1}^{2}-1}$ and $\beta_{2 d}=\beta_{2} \sqrt{1-\varsigma_{2}^{2}}$, depending upon the values of $\varsigma_{i}$. Using the above analysis and employing the inverse of the Laplace transform in (4.11) and (4.12), sway-roll-yaw motions are obtained as

$$
\begin{aligned}
& \eta_{2}(t)=\left(\frac{1}{g_{4}}\right)\left[\alpha_{1}+\alpha_{2} \cos \varpi t+\left(\frac{\alpha_{3}}{\varpi}\right) \sin \varpi t+\alpha_{4} e^{-\varsigma_{1} \beta_{1} t} \cosh \left(\beta_{1} \sqrt{\varsigma_{1}^{2}-1}\right) t\right. \\
& +\left(\alpha_{5}-\alpha_{4} \varsigma_{1} \beta_{1}\right) e^{-\varsigma_{1} \beta_{1} t} \frac{\sinh \left(\beta_{1} \sqrt{\varsigma_{1}^{2}-1}\right) t}{\beta_{1} \sqrt{\varsigma_{1}^{2}-1}} \\
& +\alpha_{6} e^{-\varsigma_{1} \beta_{1} t} \cos \left(\beta_{2} \sqrt{1-\varsigma_{2}^{2}}\right) t \\
& \left.+\left(\alpha_{7}-\alpha_{6} \varsigma_{2} \beta_{2}\right) e^{-\varsigma_{2} \beta_{2} t} \frac{\sin \left(\beta_{2} \sqrt{1-\varsigma_{2}^{2}}\right) t}{\beta_{2} \sqrt{1-\varsigma_{2}^{2}}}\right], \\
& \eta_{4}(t)=\left(\frac{1}{g_{4}}\right)\left[\alpha_{1}^{\prime} \cos \varpi t+\left(\frac{\alpha_{2}^{\prime}}{\varpi}\right) \sin \varpi t\right. \\
& +\alpha_{3}^{\prime} e^{-\varsigma_{1} \beta_{1} t} \cosh \left(\beta_{1} \sqrt{\varsigma_{1}^{2}-1}\right) t \\
& +\left(\alpha_{4}^{\prime}-\alpha_{3}^{\prime} \varsigma_{1} \beta_{1}\right) e^{-\varsigma_{1} \beta_{1} t} \frac{\sinh \left(\beta_{1} \sqrt{\varsigma_{1}^{2}-1}\right) t}{\beta_{1} \sqrt{\varsigma_{1}^{2}-1}}
\end{aligned}
$$




$$
\begin{aligned}
& +\alpha_{5}^{\prime} e^{-\varsigma_{2} \beta_{2} t} \cos \left(\beta_{2} \sqrt{1-\varsigma_{2}^{2}}\right) t \\
& \left.+\left(\alpha_{6}^{\prime}-\alpha_{5}^{\prime} \varsigma_{2} \beta_{2}\right) e^{-\varsigma_{2} \beta_{2} t} \frac{\sin \left(\beta_{2} \sqrt{1-\varsigma_{2}^{2}}\right) t}{\beta_{2} \sqrt{1-\varsigma_{2}^{2}}}\right], \\
& \eta_{6}(t)=\left(\frac{1}{g_{4}}\right)\left[\alpha_{1}^{\prime \prime}+\alpha_{2}^{\prime \prime} \cos \varpi t+\left(\frac{\alpha_{3}^{\prime \prime}}{\varpi}\right) \sin \varpi t+\alpha_{4}^{\prime \prime} e^{-\varsigma_{1} \beta_{1} t} \cosh \left(\beta_{1} \sqrt{\varsigma_{1}^{2}-1}\right) t\right. \\
& +\left(\alpha_{5}^{\prime \prime}-\alpha_{4}^{\prime \prime} \varsigma_{1} \beta_{1}\right) e^{-\varsigma_{1} \beta_{1} t} \frac{\sinh \left(\beta_{1} \sqrt{\varsigma_{1}^{2}-1}\right) t}{\beta_{1} \sqrt{\varsigma_{1}^{2}-1}} \\
& +\alpha_{6}^{\prime \prime} e^{-\varsigma_{1} \beta_{1} t} \cos \left(\beta_{2} \sqrt{1-\varsigma_{2}^{2}}\right) t \\
& \left.+\left(\alpha_{7}^{\prime \prime}-\alpha_{6}^{\prime \prime} \varsigma_{2} \beta_{2}\right) e^{-\varsigma_{2} \beta_{2} t} \frac{\sin \left(\beta_{2} \sqrt{1-\varsigma_{2}^{2}}\right) t}{\beta_{2} \sqrt{1-\varsigma_{2}^{2}}}\right] .
\end{aligned}
$$

7. Results and discussion. To illustrate the floating body motions, the exact solutions obtained in (6.1), (6.2), and (6.3) are evaluated numerically. The unknown coefficients which appear in the above expressions are determined by using the Gauss elimination method prior to applying the Laplace inverse. The detailed description of the entire procedure is given in Figure 5.1. A close inspection of the analytical solution reveals that the expressions (6.1) and (6.3) are similar in nature. This is due to the absence of restoring forces in sway and yaw equations of motion. However, the governing equation for roll contains hydrostatic restoring force inherently and, as a consequence, roll motion manifests harmonic behavior. In the absence of wave force, sway and yaw motions do not preserve harmonic property. Under the action of sinusoidal waves, the solution obtained for the three modes of motion are grouped into three parts; (i) constant term, indicating shift (ii) oscillatory term, indicating harmonic behavior, and (iii) decay term. In roll motion, the constant term representing shift is absent.

The time history of forcing function (wave force) and sway motions for frequencies $0.56 \mathrm{rad} / \mathrm{s}, 0.74 \mathrm{rad} / \mathrm{s}$, and $1.24 \mathrm{rad} / \mathrm{s}$ are shown in Figures $7.1 \mathrm{a}$ and $7.1 \mathrm{~b}$. The wave periods corresponding to the three frequencies are obtained as 11.2, 8.5, and 5.1 seconds. It can be observed that the sway displacement is maximum for wave frequency $0.56 \mathrm{rad} / \mathrm{s}$ and it decreases as wave frequency increases. The maximum sway displacement corresponding to these frequencies are found to be $65 \mathrm{~m}, 9 \mathrm{~m}$, and $1.75 \mathrm{~m}$, respectively. For all three frequencies, sway motions become harmonic with the elapse of time $t>100$. In (6.1), the terms having a factor $e^{-\zeta_{i} \beta_{i} t}$ indicate sway damping, which eventually dies out as $t \rightarrow \infty$. The time required for attainment of dynamic equilibrium corresponding to these frequencies is found to be 100,50 , and 35 seconds, respectively.

Figures $7.2 \mathrm{a}$ and $7.2 \mathrm{~b}$ show the forcing function and the time history of roll displacements (in degree) measured in the anticlockwise direction with respect to the $x$-axis. As the floating body is initially at rest, the effect of damping on oscillation is noticed for $t<50$ seconds. As $t \rightarrow \infty$, the effect of damping ceases and, consequently, the first and 


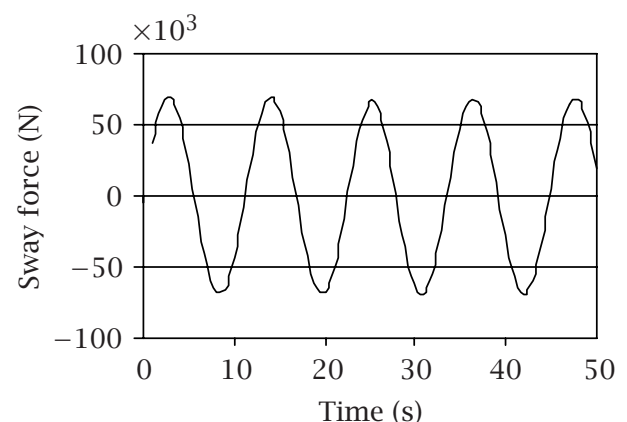

(a)

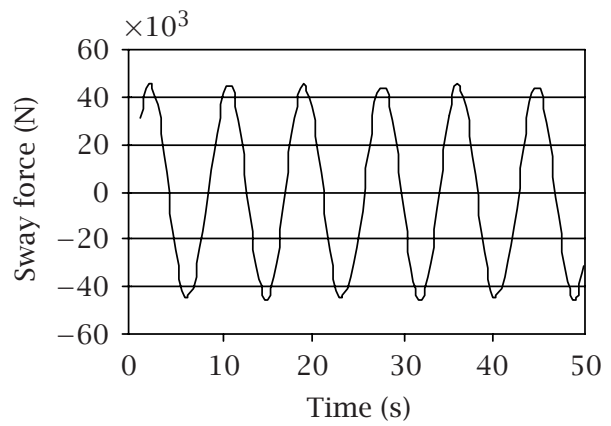

(c)

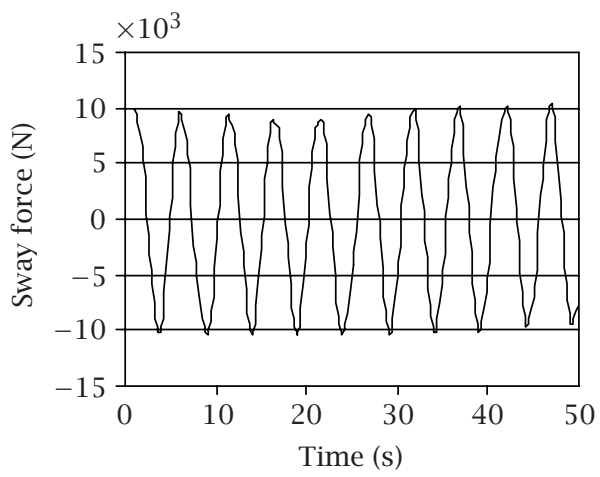

(e)

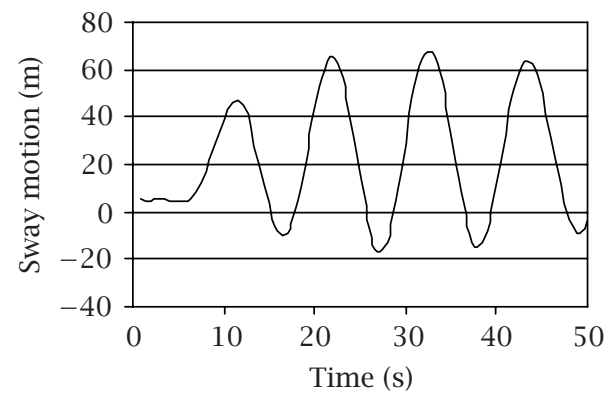

(b)

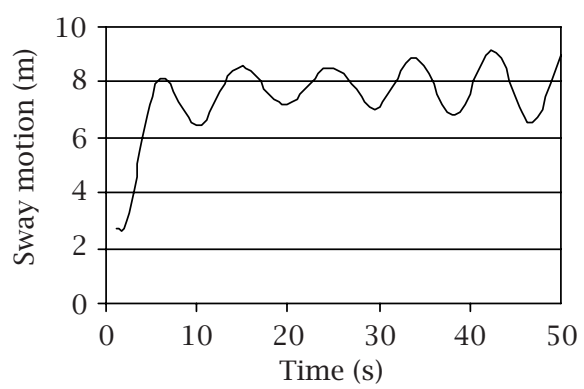

(d)

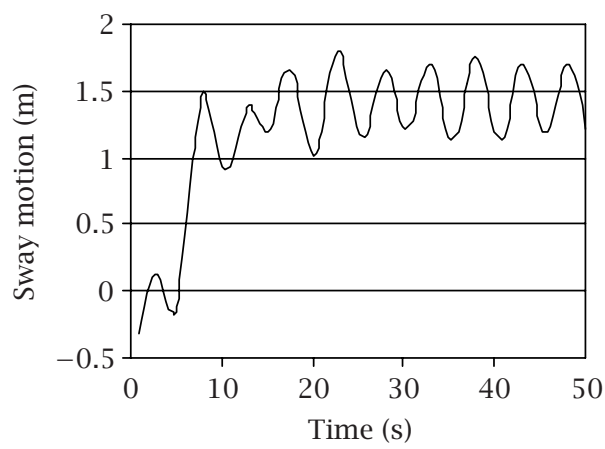

(f)

Figure 7.1. (a, c, and e) Time history of forcing function and (b, d, and f) sway motion for wave frequencies $(\mathrm{a}, \mathrm{b}) 0.56 \mathrm{rad} / \mathrm{s}$, (c, d) $0.74 \mathrm{rad} / \mathrm{s}$, and (e, f) $1.24 \mathrm{rad} / \mathrm{s}$. 


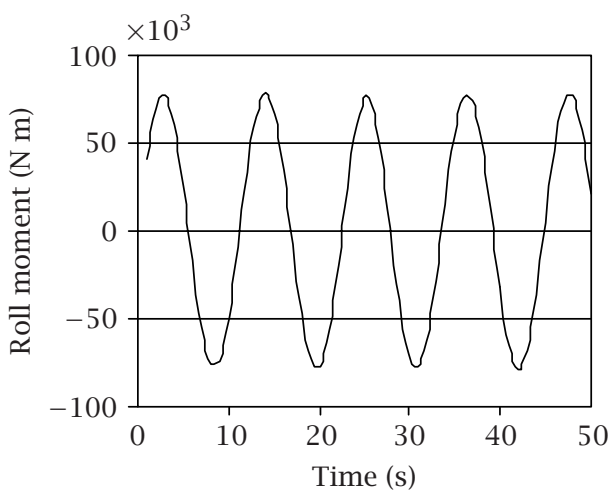

(a)

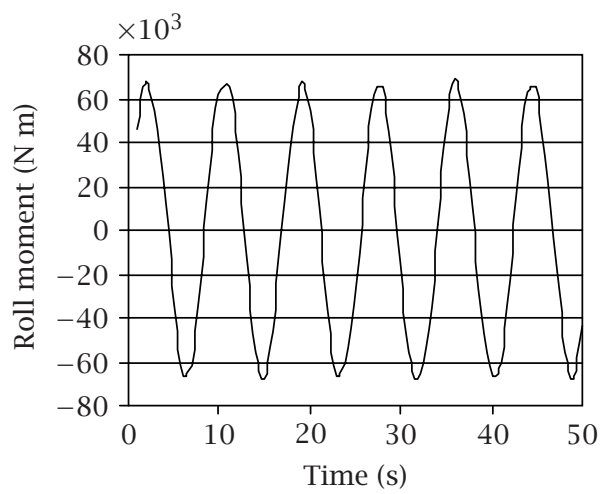

(c)

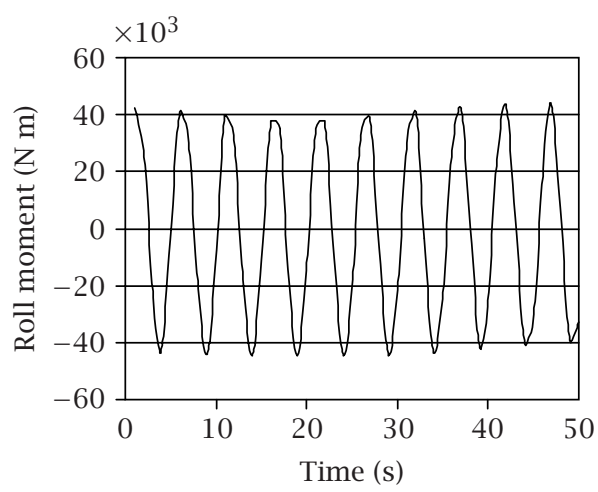

(e)

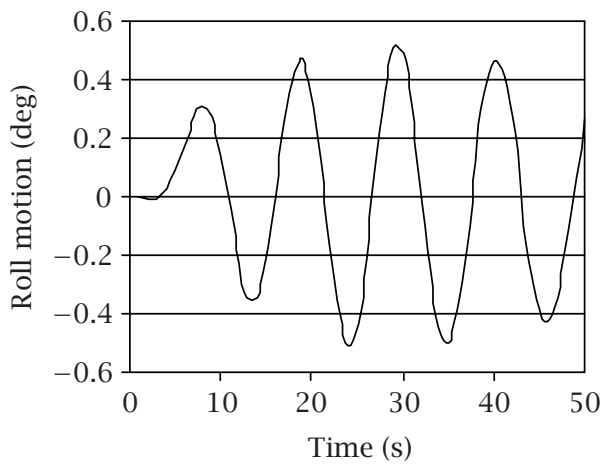

(b)

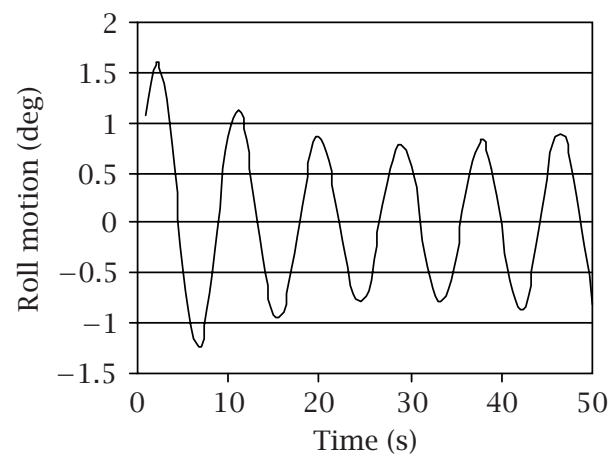

(d)

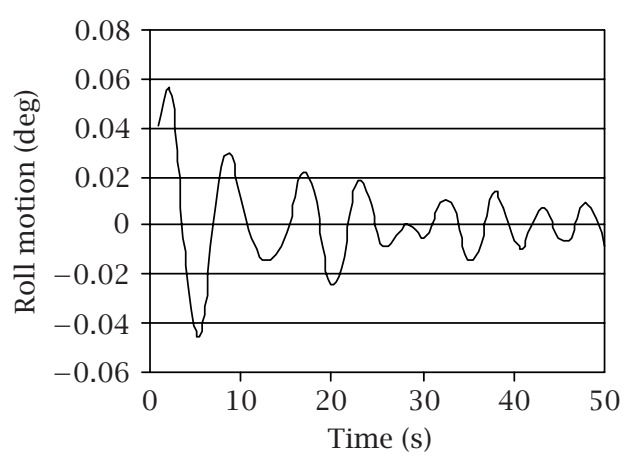

(f)

Figure 7.2. (a, c, and e) Time history of forcing function and (b, d, f) roll motion for wave frequencies $(\mathrm{a}, \mathrm{b}) 0.56 \mathrm{rad} / \mathrm{s},(\mathrm{c}, \mathrm{d}) 0.74 \mathrm{rad} / \mathrm{s}$, and $(\mathrm{e}, \mathrm{f})$ $1.24 \mathrm{rad} / \mathrm{s}$. 


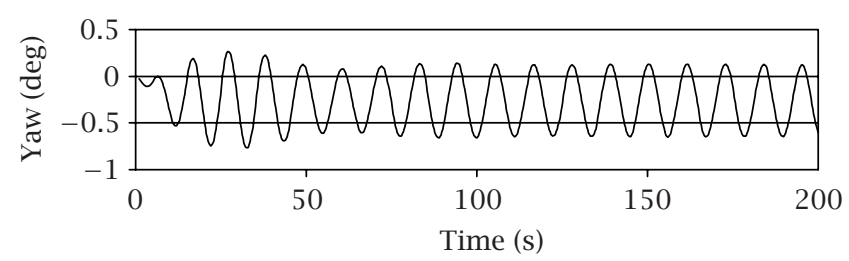

(a)

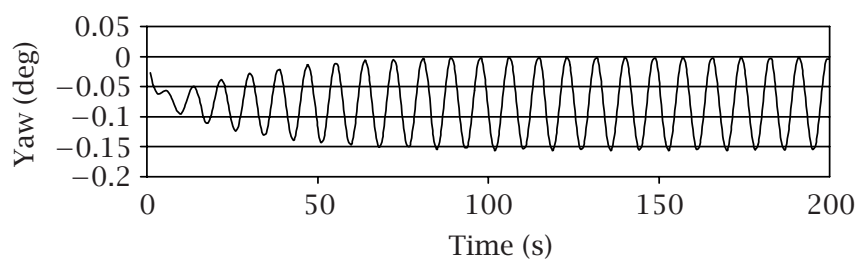

(b)

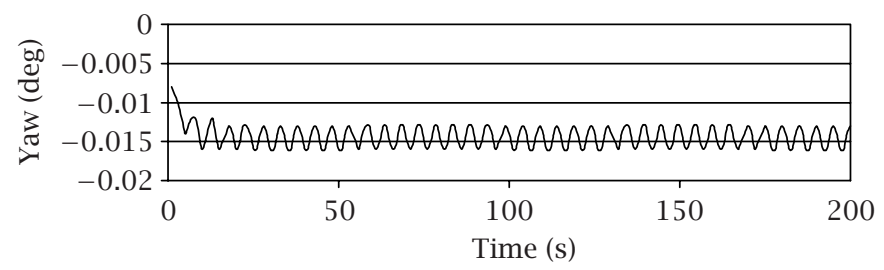

(c)

FIGURE 7.3. Time history of yaw motion for wave frequencies: (a) $0.56 \mathrm{rad} / \mathrm{s}$, (b) $0.74 \mathrm{rad} / \mathrm{s}$, and (c) $1.24 \mathrm{rad} / \mathrm{s}$.

second terms of (6.2) only contribute. Subsequently, roll motions for all three frequencies show harmonic behavior for $t>50$ seconds. The maximum roll displacements for wave frequencies $0.56 \mathrm{rad} / \mathrm{s}, 0.74 \mathrm{rad} / \mathrm{s}$, and $1.24 \mathrm{rad} / \mathrm{s}$ are $\pm 0.5^{\circ}, \pm 1.6^{\circ}$, and $\pm 0.05^{\circ}$, respectively.

The yaw motions (in degree) measured in the anticlockwise direction with respect to the $z$-axis show the angular displacements for all three frequencies (Figure 7.3). The predominant yaw angles are found to be negative, and their frequency-wise maximum values are obtained as $-0.75^{\circ},-0.15^{\circ}$, and $-0.016^{\circ}$, respectively, while subjected to the same wave forces. This is due to the fact that the location of the center of gravity of the floating body is $-1 \mathrm{~m}$ away from the origin of the $x$-axis, which creates uneven yaw moments. The amplitude of yaw angles decreases with the increase of wave frequency. 
Appendix. Expressions for added-mass coefficients and damping coefficients (with zero forward speed) (derived from Salvesen et al. [4]) which appeared in (2.3) are as follows:

$$
\begin{aligned}
& A_{22}=\int a_{22} d \xi, \quad A_{24}=A_{42}=\int a_{24} d \xi, \quad A_{26}=\int \xi a_{22} d \xi, \quad A_{44}=\int a_{44} d \xi, \\
& A_{46}=\int \xi a_{24} d \xi, \quad A_{62}=A_{26}, \quad A_{64}=A_{46}, \quad A_{66}=\int \xi^{2} a_{22} d \xi, \\
& B_{22}=\int b_{22} d \xi, \quad B_{24}=\int b_{24} d \xi=B_{42}, \quad B_{26}=\int \xi b_{22} d \xi, \quad B_{44}=\int b_{44} d \xi, \\
& B_{46}=\int \xi b_{24} d \xi, \quad B_{62}=B_{26}, \quad B_{64}=B_{46}, \quad B_{66}=\int \xi^{2} b_{22} d \xi,
\end{aligned}
$$

where $a_{j k}$ denote two-dimensional sectional added-mass coefficients and $b_{j k}$ denote two-dimensional sectional damping coefficients. Steps involved in the expansion of determinant $D$, which appeared in (4.4), are as in (4.6), where

$$
\begin{array}{lll}
l_{1}=s^{2}+a_{2} s, & m_{1}=b_{1} s^{2}+b_{2} s, & n_{1}=c_{1} s^{2}+c_{2} s, \\
l_{2}=a_{4} s^{2}+a_{5} s, & m_{2}=s^{2}+b_{5} s+b_{6}, & n_{2}=c_{4} s^{2}+c_{5} s, \\
l_{3}=a_{7} s^{2}+a_{8} s, & m_{3}=b_{7} s^{2}+b_{8} s, & n_{3}=s^{2}+c_{8} s .
\end{array}
$$

Substitution of $l_{i}, m_{i}$, and $n_{i}(i=1,2,3)$ in $D$ leads to

$$
D=s^{2}\left(g_{4} s^{4}+g_{3} s^{3}+g_{2} s^{2}+g_{1} s+g_{0}\right)
$$

where

$$
\begin{aligned}
g_{4}= & \left(1-b_{7} c_{4}\right)+a_{4}\left(b_{7} c_{1}-b_{1}\right)+a_{7}\left(b_{1} c_{4}-c_{1}\right), \\
g_{3}= & \left(b_{5}+c_{8}-b_{8} c_{4}-b_{7} c_{5}+a_{2}-a_{2} b_{7} c_{4}\right)+a_{4}\left(b_{7} c_{2}+b_{8} c_{1}-b_{2}-b_{1} c_{8}\right) \\
& +a_{5}\left(b_{7} c_{1}-b_{1}\right)+a_{7}\left(b_{1} c_{5}+b_{2} c_{4}\right)-a_{7}\left(b_{5} c_{1}+c_{2}\right)+a_{8}\left(b_{1} c_{4}-c_{1}\right), \\
g_{2}= & \left(b_{6}+b_{5} c_{8}-b_{8} c_{5}+a_{2} b_{5}+a_{2} c_{8}-a_{2} b_{8} c_{4}-a_{2} b_{7} c_{5}\right) \\
& +a_{4}\left(b_{8} c_{2}-b_{2} c_{8}\right)+c_{5}\left(b_{7} c_{2}+b_{8} c_{1}-b_{2}-b_{1} c_{8}\right)+a_{7} b_{2} c_{5}-a_{7}\left(b_{6} c_{1}+b_{5} c_{2}\right) \\
& +a_{8}\left(b_{1} c_{5}+b_{2} c_{4}\right)-a_{8}\left(b_{5} c_{1}+c_{2}\right), \\
g_{1}= & \left(b_{6} c_{8}+a_{2} b_{6}+a_{2} b_{5} c_{8}-a_{2} b_{8} c_{5}\right)+a_{5}\left(b_{8} c_{2}-b_{2} c_{8}\right)+a_{8} b_{2} c_{5}-a_{8}\left(b_{6} c_{1}+b_{5} c_{2}\right)-a_{7} b_{6} c_{2}, \\
g_{0}= & a_{2} b_{6} c_{8}-a_{8} b_{6} c_{2}, \\
D= & s^{2} g_{4}\left(s^{4}+r_{3} s^{3}+r_{2} s^{2}+r_{1} s+r_{0}\right),
\end{aligned}
$$

where

$$
\begin{aligned}
r_{3} & =\frac{g_{3}}{g_{4}}, \quad r_{2}=\frac{g_{2}}{g_{4}}, \quad r_{1}=\frac{g_{1}}{g_{4}}, \quad r_{0}=\frac{g_{0}}{g_{4}}, \\
D & =s^{2} g_{4}\left(s^{2}+u_{1} s+u_{0}\right)\left(s^{2}+v_{1} s+v_{0}\right) \\
& =s^{2} g_{4}\left(s^{2}+2 \varsigma_{1} \beta_{1} s+\beta_{1}^{2}\right)\left(s^{2}+2 \varsigma_{2} \beta_{2} s+\beta_{2}^{2}\right),
\end{aligned}
$$


where

$$
\beta_{1}=\sqrt{u_{0}}, \quad \varsigma_{1}=\frac{u_{1}}{2 \sqrt{u_{0}}}, \quad \beta_{2}=\sqrt{\nu_{0}}, \quad \varsigma_{2}=\frac{v_{1}}{2 \sqrt{\nu_{0}}}
$$

A quartic can be considered as the product of two quadratic factors. Therefore, we write

$$
s^{4}+r_{3} s^{3}+r_{2} s^{2}+r_{1} s^{1}+r_{0}=\left(s^{2}+u_{1} s+u_{0}\right)\left(s^{2}+v_{1} s+v_{0}\right)
$$

then

$$
\begin{gathered}
u_{1}+v_{1}=r_{3}, \\
u_{0}+v_{0}+u_{1} v_{1}=r_{2}, \\
u_{1} v_{0}+u_{0} v_{1}=r_{1}, \\
u_{0} v_{0}=r_{0} .
\end{gathered}
$$

If (A.8) and (A.10) are solved simultaneously in order to obtain $u_{1}$ in terms of $u_{0}$ and $v_{0}$, (A.8), (A.9), (A.10), and (A.11) can be replaced with (A.12), (A.13), (A.14), and (A.15) as follows:

$$
\begin{gathered}
u_{0} v_{0}=r_{0}, \\
u_{1}=\frac{r_{1}-u_{0} r_{3}}{v_{0}-u_{0}}, \\
v_{1}=r_{3}-u_{1}, \\
{[2 p t] u_{0}+v_{0}+u_{1} v_{1}=r_{2} .}
\end{gathered}
$$

Now a trial-and-error solution is applied. Assuming $v_{0}$, we calculate $u_{0}$ from (A.12), $u_{1}$ from (A.13), $v_{1}$ from (A.14), and check whether (A.15) is satisfied. In this process, it is possible that $u_{0}$ could be equal to $v_{0}$ so that $u_{1}$ need not be calculated from (A.13). Hence, a new set of equations may be used:

$$
\begin{gathered}
u_{0}=v_{0}, \\
\frac{r_{1}}{r_{3}}=u_{0}, \\
r_{3}^{2}+8 u_{0} \geq 4 r_{2}, \\
u_{1}=r_{3}+\frac{\sqrt{r_{3}^{2}-4\left(r_{2}-2 r_{1} / r_{3}\right)}}{2}, \\
v_{1}=r_{3}-u_{1} .
\end{gathered}
$$

Steps involved in the expansion of $D_{1}$ are as follows:

$$
D_{1}=r_{1}\left(m_{2} n_{3}-n_{2} m_{3}\right)+r_{2}\left(m_{3} n_{1}-n_{3} m_{1}\right)+r_{3}\left(m_{1} n_{2}-n_{1} m_{2}\right),
$$


where

$$
\begin{aligned}
& r_{1}=p_{1}+q_{1}, \\
& p_{1}=\bar{p}_{1}^{1} s+\bar{p}_{0}^{1}, \\
& \bar{p}_{1}^{1}=\left\{\eta_{2}(0)+b_{1} \eta_{4}(0)+c_{1} \eta_{6}(0)\right\}, \\
& \bar{p}_{0}^{1}=\left\{a_{2} \eta_{2}(0)+b_{2} \eta_{4}(0)+c_{2} \eta_{6}(0)+\dot{\eta}_{2}(0)+b_{1} \dot{\eta}_{4}(0)+c_{1} \dot{\eta}_{6}(0)\right\}, \\
& q_{1}=\frac{K_{2} \varpi}{s^{2}+\varpi^{2}}, \\
& r_{1}=p_{1}+q_{1}=\frac{\sum_{i=0}^{3} \bar{r}_{i}^{1} s^{i}}{s^{2}+\varpi^{2}} \\
& \bar{r}_{3}^{1}=\bar{p}_{1}^{1}, \quad \bar{r}_{2}^{1}=\bar{p}_{0}^{1}, \quad \bar{r}_{1}^{1}=\bar{p}_{1}^{1} \varpi^{2}, \quad \bar{r}_{0}^{1}=\bar{p}_{0}^{1} \varpi^{2}+K_{2} \varpi, \\
& r_{2}=p_{2}+q_{2} \text {, } \\
& p_{2}=\bar{p}_{1}^{2} s+\bar{p}_{0}^{2}, \quad \bar{p}_{1}^{2}=\left\{a_{4} \eta_{2}(0)+\eta_{4}(0)+c_{4} \eta_{6}(0)\right\}, \\
& \bar{p}_{0}^{2}=\left\{a_{5} \eta_{2}(0)+b_{5} \eta_{4}(0)+c_{5} \eta_{6}(0)+a_{4} \dot{\eta}_{2}(0)+\dot{\eta}_{4}(0)+c_{4} \dot{\eta}_{6}(0)\right\}, \\
& q_{2}=\frac{K_{4} \varpi}{s^{2}+\varpi^{2}}, \\
& r_{2}=p_{2}+q_{2}=\frac{\sum_{i=0}^{3} \bar{r}_{i}^{2} s^{i}}{s^{2}+\varpi^{2}} \\
& \bar{r}_{3}^{2}=\bar{p}_{1}^{2}, \quad \bar{r}_{2}^{2}=\bar{p}_{0}^{2}, \quad \bar{r}_{1}^{2}=\bar{p}_{1}^{2} \varpi^{2}, \quad \bar{r}_{0}^{2}=\bar{p}_{0}^{2} \varpi_{1}^{2}+K_{4} \varpi, \\
& r_{3}=p_{3}+q_{3}, \\
& p_{3}=\bar{p}_{1}^{3} s+\bar{p}_{0}^{3}, \quad \bar{p}_{1}^{3}=\left\{a_{7} \eta_{2}(0)+b_{7} \eta_{4}(0)+\eta_{6}(0)\right\}, \\
& \bar{p}_{0}^{3}=\left\{a_{8} \eta_{2}(0)+b_{8} \eta_{4}(0)+c_{8} \eta_{6}(0)+a_{7} \dot{\eta}_{2}(0)+b_{7} \dot{\eta}_{4}(0)+\dot{\eta}_{6}(0)\right\}, \\
& q_{3}=\frac{K_{6} \varpi}{s^{2}+\varpi^{2}}, \\
& r_{3}=p_{3}+q_{3}=\frac{\sum_{i=0}^{3} \bar{r}_{i}^{3} s^{i}}{s^{2}+\varpi^{2}}, \\
& \bar{r}_{3}^{3}=\bar{p}_{1}^{3}, \quad \bar{r}_{2}^{3}=\bar{p}_{0}^{3}, \quad \bar{r}_{1}^{3}=\bar{p}_{1}^{3} \varpi^{2}, \quad \bar{r}_{0}^{3}=\bar{p}_{0}^{3} \varpi^{2}+K_{6} \varpi, \\
& m_{2} n_{3}-n_{2} m_{3}=s\left(\sum_{i=0}^{3} d_{i}^{1} s^{i}\right), \\
& d_{3}^{1}=1-b_{7} c_{4}, \quad d_{2}^{1}=b_{5}+c_{8}-\left(b_{8} c_{4}+b_{7} c_{5}\right), \quad d_{1}^{1}=b_{6}+b_{5} c_{8}-b_{8} c_{5}, \quad d_{0}^{1}=b_{6} c_{8}, \\
& m_{3} n_{1}-n_{3} m_{1}=s^{2}\left(\sum_{i=0}^{2} d_{i}^{2} s^{i}\right), \\
& d_{2}^{2}=b_{7} c_{1}-b_{1}, \quad d_{1}^{2}=b_{7} c_{2}+b_{8} c_{1}-\left(b_{2}+b_{1} c_{8}\right), \quad d_{0}^{2}=b_{8} c_{2}-b_{2} c_{8}, \\
& m_{1} n_{2}-n_{1} m_{2}=s\left(\sum_{i=0}^{3} d_{i}^{3} s^{i}\right),
\end{aligned}
$$




$$
\begin{gathered}
d_{3}^{3}=b_{1} c_{4}-c_{1}, \quad d_{2}^{3}=b_{1} c_{5}+b_{2} c_{4}-c_{1} b_{5}-c_{2}, \quad d_{1}^{3}=b_{2} c_{5}-b_{6} c_{1}-b_{5} c_{2}, \quad d_{0}^{3}=-b_{6} c_{2}, \\
D_{1}=\frac{1}{\left(s^{2}+\varpi^{2}\right)}\left[\left(\sum_{i=0}^{3} \bar{r}_{i}^{1} s^{i}\right) s\left(\sum_{i=0}^{3} d_{i}^{1} s^{i}\right)+\left(\sum_{i=0}^{3} \bar{r}_{i}^{2} s^{i}\right) s^{2}\left(\sum_{i=0}^{2} d_{i}^{2} s^{i}\right)+\left(\sum_{i=0}^{3} \bar{r}_{i}^{3} s^{i}\right) s\left(\sum_{i=0}^{3} d_{i}^{3} s^{i}\right)\right], \\
D_{1}=\frac{s}{\left(s^{2}+\varpi^{2}\right)}\left[\left(\sum_{i=0}^{6} \delta_{i}^{1} s^{i}\right)+s\left(\sum_{i=0}^{5} \delta_{i}^{2} s^{i}\right)+\left(\sum_{i=0}^{6} \delta_{i}^{3} s^{i}\right)\right], \\
D_{1}=\frac{s \sum_{i=0}^{6} D_{i}^{1} s^{i}}{\left(s^{2}+\varpi^{2}\right)} .
\end{gathered}
$$

Steps involved in the computation of $f_{2}(s)$ are as follows:

$$
\begin{aligned}
f_{2}(s)=\frac{D_{1}}{D} & =\frac{1}{g_{4}}\left[\frac{s \sum_{i=0}^{6} D_{i}^{1} s^{i}}{\left(s^{2}+\varpi^{2}\right) s^{2}\left(s^{2}+u_{1} s+u_{0}\right)\left(s^{2}+v_{1} s+v_{0}\right)}\right] \\
& =\frac{1}{g_{4}}\left[\frac{\alpha_{1}}{s}+\frac{\alpha_{2} s+\alpha_{3}}{s^{2}+\varpi^{2}}+\frac{\alpha_{4} s+\alpha_{5}}{s^{2}+u_{1} s+u_{0}}+\frac{\alpha_{6} s+\alpha_{7}}{s^{2}+\nu_{1} s+v_{0}}\right],
\end{aligned}
$$

where $\alpha_{i} s$ are computed with the help of the Gauss elimination method from the set of seven equations as follows:

$$
\sum_{j=1}^{7} \bar{a}_{i j} \alpha_{j}=d_{i}, \quad i=1,2, \ldots, 7,
$$

where

$$
\begin{aligned}
& \bar{a}_{11}=\varpi^{2} r_{0}, \quad \bar{a}_{12}=0, \quad \bar{a}_{13}=0, \quad \bar{a}_{14}=0, \quad \bar{a}_{15}=0, \quad \bar{a}_{16}=0, \quad \bar{a}_{17}=0, \\
& \bar{a}_{21}=\varpi^{2} r_{1}, \quad \bar{a}_{22}=0, \quad \bar{a}_{23}=r_{0}, \quad \bar{a}_{24}=0, \\
& \bar{a}_{25}=\varpi^{2} v_{0}, \quad \bar{a}_{26}=0, \quad \bar{a}_{27}=\varpi^{2} u_{0}, \\
& \bar{a}_{31}=r_{0}+\varpi^{2} r_{2}, \quad \bar{a}_{32}=r_{0}, \quad \bar{a}_{33}=r_{1}, \\
& \bar{a}_{34}=\varpi^{2} v_{0}, \quad \bar{a}_{35}=\varpi^{2} v_{1}, \quad \bar{a}_{37}=\varpi^{2} u_{1}, \\
& \bar{a}_{41}=r_{1}+\varpi^{2} r_{3}, \quad \bar{a}_{42}=r_{1}, \quad \bar{a}_{43}=r_{2}, \quad \bar{a}_{44}=\varpi^{2} v_{1}, \\
& \bar{a}_{45}=v_{0}+\varpi^{2}, \quad \bar{a}_{46}=\varpi^{2} u_{1}, \quad \bar{a}_{47}=u_{0}+\varpi^{2}, \\
& \bar{a}_{51}=r_{1}, \quad \bar{a}_{52}=r_{2}+\varpi^{2}, \quad \bar{a}_{53}=r_{3}, \quad \bar{a}_{54}=v_{0}+\varpi^{2}, \\
& \bar{a}_{55}=v_{1}, \quad \bar{a}_{56}=u_{0}+\varpi^{2}, \quad \bar{a}_{57}=u_{10}, \quad \bar{a}_{63}=1, \quad \bar{a}_{64}=v_{1}, \quad \bar{a}_{65}=1, \quad \bar{a}_{66}=u_{1}, \quad \bar{a}_{67}=1, \\
& \bar{a}_{61}=r_{3}, \quad \bar{a}_{62}=r_{3}, \quad \bar{a}_{73}=0, \quad \bar{a}_{74}=1, \quad \bar{a}_{75}=0, \quad \bar{a}_{76}=1, \quad \bar{a}_{77}=0, \\
& \bar{a}_{71}=1, \quad \bar{a}_{72}=1, \quad \bar{a}_{73}=1, \quad d_{3}, \quad d_{6}=D_{5}^{1}, \quad d_{7}=D_{6}^{1} .
\end{aligned}
$$

In a similar manner, computations were carried out for $f_{4}(s)$ and $f_{6}(s)$. These are being omitted here for briefness. 
ACKNOWLeDgement. The first author is thankful to Mrs. V. M. Bendre, Director of Central Water and Power Research Station, Pune, India, for giving permission to communicate this paper.

\section{REFERENCES}

[1] W. F. Cummins, The impulse response function and ship motions, Schiffstechnik 9 (1962), 101-109.

[2] O. M. Faltinsen, J. N. Newman, and T. Vinje, Nonlinear wave loads on a slender vertical cylinder, J. Fluid Mech. 289 (1995), 179-198

[3] M. Mulk and J. Falzarano, Complete 6-degrees-of-freedom nonlinear ship rolling motion, J. Offshore Mechanics and Arctic Engineering 116 (1994), no. 4, 191-201.

[4] N. Salvesen, E. O. Tuck, and O. M. Faltinsen, Ship motions and sea loads, Trans. SNAME 78 (1970), 250-287.

[5] S. Surendran and J. V. R. Reddy, Numerical simulation of ship stability for dynamic environment, Ocean Engineering 30 (2003), no. 10, 1305-1317.

[6] F. Tasai, On the swaying, yawing and rolling motions of ships in oblique waves, International Shipbuilding Progress 14 (1967), no. 153, 216-228.

[7] M. Taylan, The effect of nonlinear damping and restoring in ship rolling, Ocean Engineering 27 (2000), no. 9, 921-932.

[8] F. Ursell, Irregular frequencies and the motion of floating bodies, J. Fluid Mech. 105 (1981), 143-156.

[9] J. H. Vugts, The hydrodynamic coefficients for swaying, heaving and rolling cylinders in a free surface, Tech. Report 194, Laboratorium voor Scheepsbouwkunde, Technische Hogeschool Delft, The Netherlands, 1968.

[10] G. Weinblum and M. St. Denis, On the motions of ships at sea, Trans. SNAME 58 (1950), 184-231.

S. N. Das: Central Water and Power Research Station, Khadakwasla, Pune-411 024, Maharashtra, India

E-mail address: wapis@mah.nic.in

S. K. Das: Institute of Applied Mechanics, National Taiwan University, Taipei 10764, Taiwan

E-mail address: sami rd@cdacindia.com 


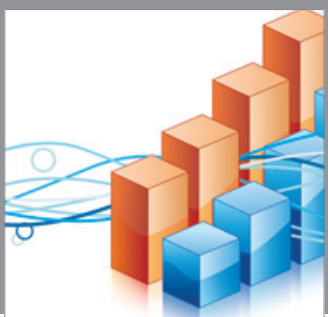

Advances in

Operations Research

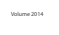

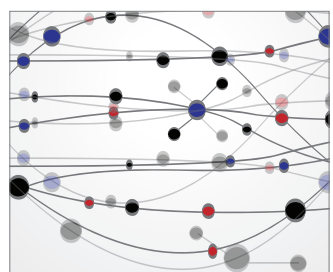

\section{The Scientific} World Journal
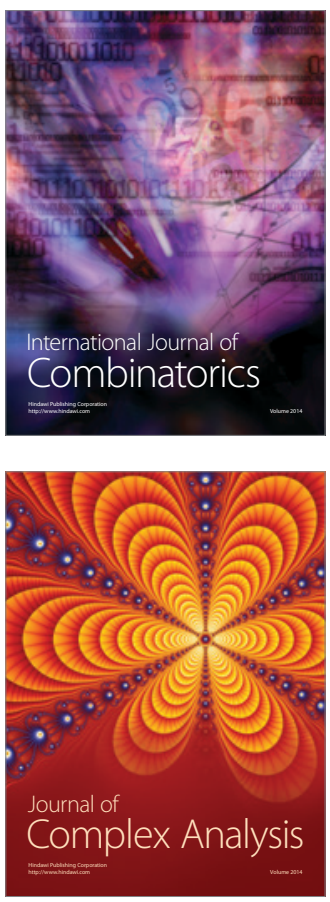

International Journal of

Mathematics and

Mathematical

Sciences
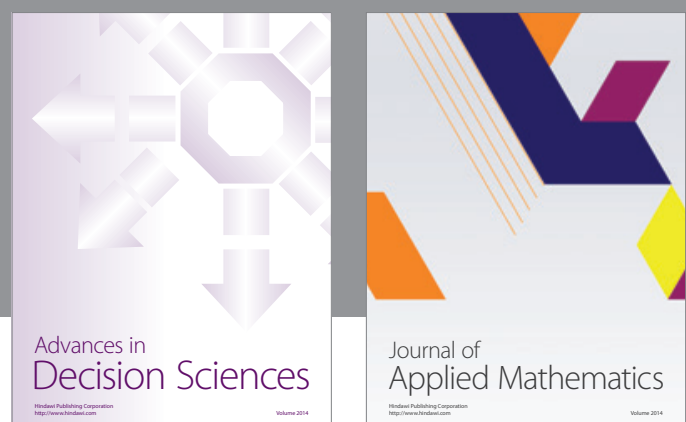

Journal of

Applied Mathematics
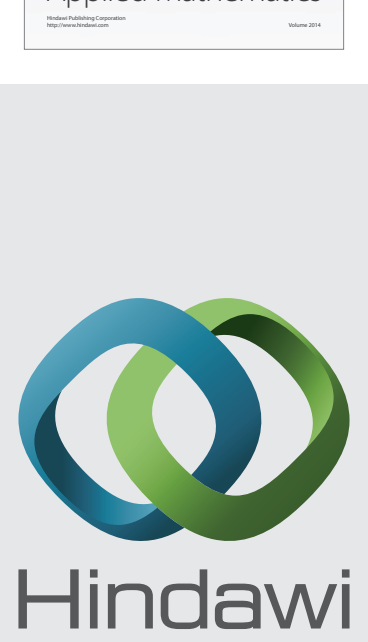

Submit your manuscripts at http://www.hindawi.com
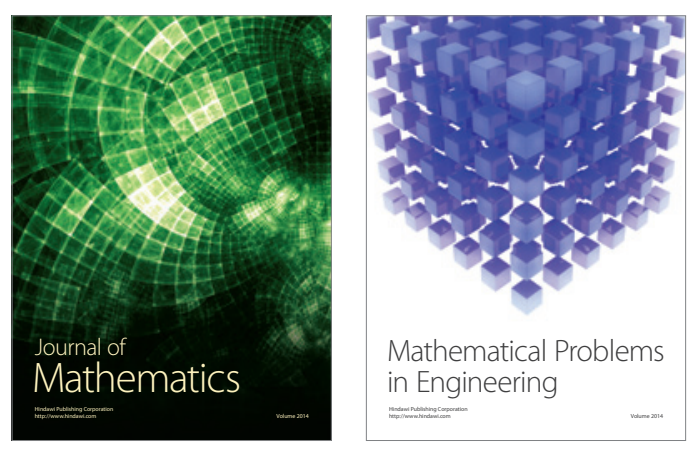

Mathematical Problems in Engineering
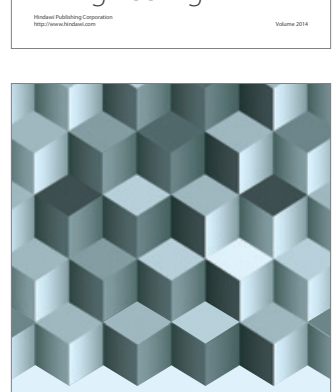

Journal of

Function Spaces
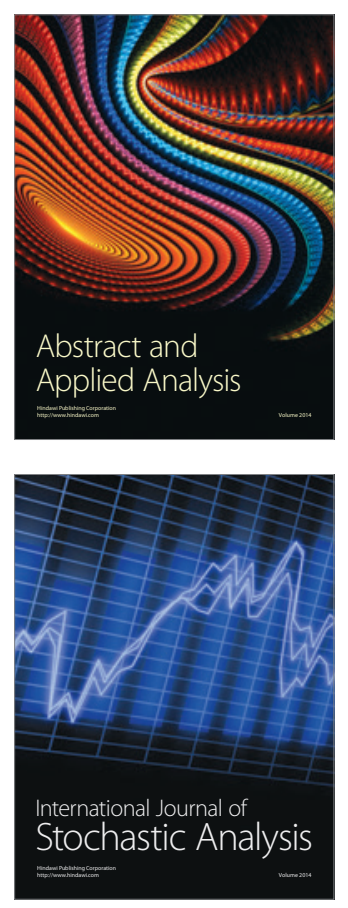

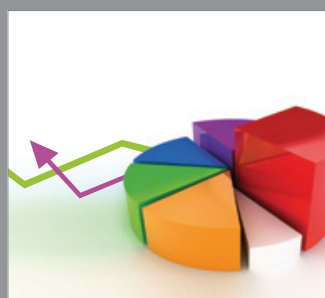

ournal of

Probability and Statistics

Promensencen
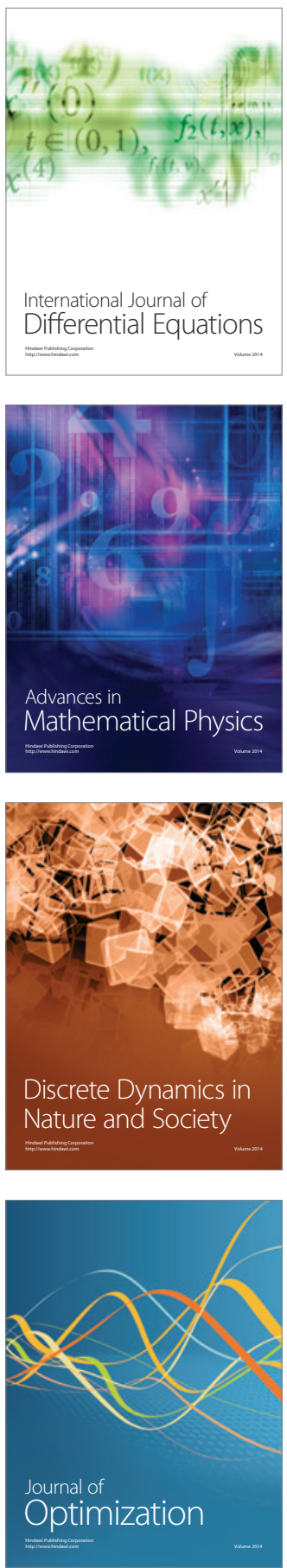